\title{
Variational Multiscale error estimators for solid mechanics adaptive simulations: an Orthogonal Subgrid Scale approach
}

\author{
Joan Baiges ${ }^{1}$ and Ramon Codina ${ }^{1,2}$ \\ ${ }^{1}$ Universitat Politècnica de Catalunya, Jordi Girona 1-3, Edifici C1, 08034 Barcelona, Spain \\ ${ }^{2}$ Centre Internacional de Mètodes Numèrics a l'Enginyeria (CIMNE), \\ Edifici C1, Campus Nord UPC C/ Gran Capità S/N 08034 Barcelona, Spain \\ joan.baiges@upc.edu, ramon.codina@upc.edu
}

July 7, 2017

\begin{abstract}
In this work we present a general error estimator for the finite element solution of solid mechanics problems based on the Variational Multiscale method. The main idea is to consider a rich model for the subgrid scales as an error estimator. The subscales are considered to belong to a space orthogonal to the finite element space (Orthogonal Subgrid Scales) and we take into account their contribution both in the element interiors and on the element boundaries (Subscales on the Element Boundaries). A simple analysis shows that the upper bound for the obtained error estimator is sharper than in other error estimators based on the Variational Multiscale Method. Numerical examples show that the proposed error estimator is an accurate approximation for the energy norm error and can be used both in simple linear constitutive models and in more complex non-linear cases.
\end{abstract}

\section{Introduction}

In the general solution of computational solid mechanics problems using finite element approximations, many times one faces situations where it is convenient to focus the computational effort in certain subdomains of interest. This is the case for instance when simulating fracture processes, where very large deformation gradients are concentrated along fracture lines. If the position and orientation of fracture lines are known a priori, a suitably refined computational mesh can be built beforehand.

However, in most engineering cases of interest the location of such failure lines is precisely part of the information that one expects to obtain from a computational simulation. As a consequence, the information required to build such meshes is not known a priori. In this case one faces with the need of either building extremely fine computational meshes capable of appropriately capturing the details of the solution no matter where these appear, or running successive simulations with different computational meshes which are progressively built as more information is available.

Adaptive mesh refinement (AMR) techniques $[1,2,3]$ appear precisely to deal with simulations where the refinement requirements are not known a priori. AMR consists in introducing local modifications in the computational mesh in such a way that the computational effort for attaining a certain error level is minimized. Or, equivalently, adaptive strategies allow one to maximize the accuracy of the obtained solution for a given computational cost.

There are two main ingredients which are involved in an AMR simulation: the first one is the actual mesh refinement algorithm, which allows one to increase the accuracy of the 
numerical solution in a given region of the computational domain. Several families of methods exist which are able to modify a given finite element mesh into a new mesh which is more convenient and results in a reduced approximation error. For the sake of conciseness, let us just remark that in our work we are focused in hierarchical $h$ refinement with hanging nodes. The treatment of hanging nodes used here consists in enforcing that the value of the unknown in these nodes is exactly the interpolated value of their parent nodes, although other possibilities for the treatment of hanging nodes such as the use of Discontinuous Galerkin approaches are possible [4], and perfectly compatible with the developments done here. Also, the proposed strategies are in principle applicable to $p$ and $r$ mesh refinement techniques, although these possibilities are not explored here. For the mesh refinement process, we rely on the adaptive refinement library RefficientLib [5,6], which is capable of running adaptively refined simulations on distributed memory parallel systems and handling meshes with hanging nodes.

The second ingredient (and the one in which this work is focused) are error estimators which are used to decide over which domain region mesh refinement is required [7, 8]. Again several families of a posteriori error estimators can be found in the literature, including explicit, implicit and goal-oriented error estimators. This work deals with the development of error estimators for AMR based on the Variational Multiscale Method (VMS) $[9,10]$.

The main idea of the VMS method is based in considering that the finite element approximation is not capable of exactly representing the solution to computational physics problems. If this is taken into account, the exact solution can be decomposed into the finite element part plus what are called the subscales, which are precisely the parts of the solution not representable by the finite element approximation. The finite element part can be resolved, but the effect of the subscales on the finite element solution (or coarse scales) needs to be modeled. Including the effect of the unresolved scales in the finite element formulation has turned out to be a very good strategy to solve many of the intrinsic drawbacks of the standard Galerkin method for finite elements: the VMS method is capable of providing stable solutions for convective problems, saddle point problems, acting as a turbulence model for fluid mechanics problems, dealing with the polution error of the Helmholtz equation and solving many other problems related to the finite element approximation of computational physics problems, see for instance the reviews in [11, 12].

Although it was already remarked in [10] that the modeling of the subgrid scales could be used as a suitable a posteriori error estimator, it has not been until much more recently that these ideas have started to be implemented. A first approach to use the VMS framework in error estimation is presented in [13], where the error norm for each element is computed for the one dimensional convection-diffusion equation. The methodology is extended to higher order elements in $[14,15]$. The previous strategy is used in two-dimensional domains in [16], where also the contribution of the error on the element boundaries is taken into account in the error estimator. The same ideas are applied to the Navier-Stokes equations in [17, 18], to linear elasticity in [19], and to higher order ordinary differential equations in [20]. Also, in [21], an algebraic subgrid scale approach is used to derive an error estimator for a mixed formulation for linear elasticity, although the contribution of the subscales in the boundary is neglected. A similar approach based on the Streamwise Upwind Petrov Galerkin (SUPG) stabilization method can be found in [22], where it is applied to the stationary convectiondiffusion equation.

A different approach can be found in [23], where following [24], the equation for the subscales is solved in a finer mesh instead of using an analytical expression for them. This idea is also pursued in [25], and applied to nearly-incompressible elasticity in [21] and to elliptic problems with highly varying physical coefficients in [26]. 
In this work a different approach is pursued in order to obtain error estimators for solid mechanics problems solved using a mixed $\boldsymbol{u}$ - $p$ formulation. The proposed error estimator is based on the Orthogonal Subgrid Scales concept presented in [27], and the model for the subscales on the element boundaries first presented in [28, 29]. This turns out in an error estimator composed of three main ingredients: the orthogonal displacement subscales in the element interiors, the displacement subscales in the element boundaries, and the pressure subscales. As it will be shown, this approach provides an elaborate model for the subscales capable of taking into account the effect of the non-resolved scales, that allows one to efficiently predict the error of the finite element approximation for mixed elasticity $\boldsymbol{u}-p$ formulations. Also, it will be shown that the subscales are capable of tracking the behavior of non-linear solid mechanics constitutive models.

The paper is organized as follows. In Section 2 the mixed $\boldsymbol{u}$-p formulation for solid mechanics is presented. Section 3 describes the proposed error estimator and its numerical properties. Numerical examples illustrating the performance of the proposed error estimator are presented in Section 4, and finally some conclusions close the paper in Section 5.

\section{Finite element formulation}

In this section we present a finite element formulation for general solid mechanics problems in the small strains context. The proposed mixed displacement - pressure $(\boldsymbol{u}-p)$ formulation is valid both for compressible materials and for materials in the incompressible limit. As it will be explained, it relies on a variational multiscale stabilizing mechanism which allows one to use arbitrary interpolation spaces for both displacement and pressure even when one is dealing with incompressible materials. The formulation departs from the equation of conservation of momentum:

$$
-\nabla \cdot \boldsymbol{\sigma}=\rho \boldsymbol{b},
$$

where $\boldsymbol{\sigma}$ represents the stress tensor, $\rho$ is the density, and $\boldsymbol{b}$ is the vector of external forces. Additionally, a constitutive equation which relates strains and stresses is required, which for linear elasticity can be stated as:

$$
\boldsymbol{\sigma}=\mathcal{C}: \boldsymbol{\epsilon}(\boldsymbol{u}) .
$$

Here $\mathcal{C}$ is the constitutive tensor, and $\boldsymbol{\epsilon}(\cdot)$ represents the symmetric gradient operator. Many times, and specially when dealing with incompressible materials, it is convenient to decompose the stress tensor into its spherical and deviatoric parts. The spherical part is then represented by means of the pressure $p$ (taken as positive in compression regime) and the deviatoric part is denoted as $\boldsymbol{\sigma}^{\prime}$ :

$$
\boldsymbol{\sigma}=-p \boldsymbol{I}+\boldsymbol{\sigma}^{\prime},
$$

where $\boldsymbol{I}$ is the identity tensor. Introducing this decomposition in the constitutive equation, we obtain:

$$
\boldsymbol{\sigma}=-p \boldsymbol{I}+\mathcal{C}^{\prime}: \boldsymbol{\epsilon}(\boldsymbol{u}),
$$

where now $\mathcal{C}^{\prime}$ represents the deviatoric part of the constitutive tensor, that is, the tensor that applied to the symmetric gradient of the displacement field yields the deviatoric part of the stresses:

$$
\mathcal{C}^{\prime}: \epsilon(\boldsymbol{u})=\sigma^{\prime} .
$$

$p$ is related to the displacement field through the following equation:

$$
p=-\kappa \nabla \cdot \boldsymbol{u},
$$


where $\kappa$ is the bulk modulus or modulus of compressibility. This yields the final form of the linear elasticity equations in $\boldsymbol{u}-p$ form: find $\boldsymbol{u}$ and $p$ such that:

$$
\begin{aligned}
-\nabla \cdot\left[\mathcal{C}^{\prime}: \boldsymbol{\epsilon}(\boldsymbol{u})\right]+\nabla p & =\rho \boldsymbol{b} \\
\nabla \cdot \boldsymbol{u}+\frac{1}{\kappa} p & =0 \quad \text { in } \Omega, \\
& \text { in } \Omega,
\end{aligned}
$$

where $\Omega$ represents the computational domain of $\mathbb{R}^{2}$ or $\mathbb{R}^{3}$. The boundary conditions for the problem are:

$$
\begin{aligned}
& \boldsymbol{u}=\overline{\boldsymbol{u}} \quad \text { on } \Gamma_{D}, \\
& \boldsymbol{\sigma} \cdot \boldsymbol{n}=\overline{\boldsymbol{t}} \quad \text { on } \Gamma_{N},
\end{aligned}
$$

where $\overline{\boldsymbol{u}}$ are the prescribed displacements in the Dirichlet boundary $\Gamma_{D}$ and $\overline{\boldsymbol{t}}$ are the prescribed tractions in the Neumann boundary $\Gamma_{N}, \boldsymbol{n}$ being the unit external normal.

Let us consider a quasi-uniform (for simplicity) finite element partition $\mathcal{T}_{h}:=\{K\}$ defined over domain $\Omega$, assumed to be polyhedral, $h$ denoting the element size. In the adaptive context this translates into considering that ratio between the largest and the smallest element of the mesh is bounded. The Galerkin weak form of the $\boldsymbol{u}$ - $p$ linear elasticity equations can be written as: find $\boldsymbol{u}_{h} \in V_{h}$ and $p_{h} \in Q_{h}$ such that:

$$
B\left(\left[\boldsymbol{u}_{h}, p_{h}\right],\left[\boldsymbol{v}_{h}, q_{h}\right]\right)=L\left(\boldsymbol{v}_{h}, q_{h}\right), \quad \forall \boldsymbol{v}_{h} \in V_{h}, \forall q_{h} \in Q_{h},
$$

with:

$$
\begin{aligned}
B\left(\left[\boldsymbol{u}_{h}, p_{h}\right],\left[\boldsymbol{v}_{h}, q_{h}\right]\right) & =\left(\boldsymbol{\epsilon}\left(\boldsymbol{v}_{h}\right), \sigma^{\prime}\left(\boldsymbol{u}_{h}\right)\right)-\left(\nabla \cdot \boldsymbol{v}_{h}, p_{h}\right)+\left(q_{h}, \nabla \cdot \boldsymbol{u}_{h}\right)+\left(q_{h}, \frac{1}{\kappa} p_{h}\right), \\
L\left(\boldsymbol{v}_{h}, q_{h}\right) & =\left\langle\boldsymbol{v}_{h}, \rho \boldsymbol{b}\right\rangle+\left\langle\boldsymbol{v}_{h}, \overline{\boldsymbol{t}}\right\rangle_{\Gamma_{N}} .
\end{aligned}
$$

Here $V_{h} \subset V=H^{1}(\Omega)$ and $Q_{h} \subset Q=L^{2}(\Omega)$ are the displacement and pressure interpolation spaces, defined on the partition $\mathcal{T}_{h}$. $(\cdot, \cdot)$ stands for the $L^{2}(\Omega)$ inner product and $\langle\cdot, \cdot\rangle_{\omega}$ for the integral of the product of two functions in a domain $\omega$, not necessarily in $L^{2}(\omega)$; the subscript is omitted when $\omega=\Omega$. Boundary conditions need to be incorporated to $V_{h}$ in the usual way.

In the incompressible limit this formulation is not stable for arbitrary spaces $V_{h}$ and $Q_{h}$, because the term $\left(q_{h}, \frac{1}{\kappa} p_{h}\right)$ tends to zero and causes the stability of the weak form to depend on a compatibility inf-sup condition between $V_{h}$ and $Q_{h}$. In order to circumvent this restriction, an additional stabilization term is introduced which ensures that the resulting linear system of equations is solvable. This additional stabilizing term can be derived by using the VMS method.

The VMS method was firstly introduced in $[9,10]$ and has since been used as a tool for stabilization and representation of multiscale phenomena in many fields. The main idea consists in decomposing the exact solution of a variational problem into the part which can be captured by the finite element space, and the part which cannot. In the problem we are considering, this translates into the following decomposition of $\boldsymbol{u}$ and $p$ :

$$
\begin{aligned}
\boldsymbol{u} & =\boldsymbol{u}_{h}+\tilde{\boldsymbol{u}} \\
p & =p_{h}+\tilde{p}
\end{aligned}
$$

where $\boldsymbol{u}_{h} \in V_{h}$ and $p_{h} \in Q_{h}$ represent the finite element parts of the solution, and $\tilde{\boldsymbol{u}} \in \tilde{V}$ and $\tilde{p} \in \tilde{Q}$ are the displacement and pressure subscales and subscale spaces, respectively. 
This decomposition leads to enhanced numerical discretization schemes, which in many cases allow one to solve the stability issues of the straightforward Galerkin variational form. In the particular case of solid mechanics problems, the use of the VMS method leads to formulations which are stable in the incompressible limit, in contrast to the standard Galerkin method which is unstable in this limit. This is the case of the formulation presented in equation (1).

Intuitively, it can be seen from the decomposition introduced in equation (2) that the subscales are a measure of the error of the obtained solution, because they represent the difference between the exact solution and the approximated one. This was originally noted in $[9,10]$, and has been exploited in several works since then. In this work, we are going to use a particular family of subgrid scales, the orthogonal subgrid scales, in order to build an error estimator for solid mechanics problems. The main particularity of this family of subscales is that it assumes that the subscales belong to spaces which are orthogonal to the finite element approximation spaces with respect to the $L^{2}(\Omega)$ inner product:

$$
\begin{aligned}
& \tilde{V}=V_{h}^{\perp}, \\
& \tilde{Q}=Q_{h}^{\perp} .
\end{aligned}
$$

The objective of this section is to obtain an accurate representation of the displacement and pressure subscales which allows us to, on the one hand, obtain a stable finite element formulation for the $\boldsymbol{u}-p$ approximation of the solid mechanics problem, and on the other, estimate the error of the finite element approximation. Let us start by introducing the decomposition (2) in the original variational form of the problem, and apply it both to the unknowns and the test functions of the problem. This allows us to separate the resulting equations into the equations for the finite element test functions, and the equations for the subscales test functions. The resulting finite element equations are:

$$
\begin{aligned}
& \left(\boldsymbol{\epsilon}\left(\boldsymbol{v}_{h}\right), \boldsymbol{\sigma}^{\prime}\left(\boldsymbol{u}_{h}\right)\right)+\left(\boldsymbol{\epsilon}\left(\boldsymbol{v}_{h}\right), \boldsymbol{\sigma}^{\prime}(\tilde{\boldsymbol{u}})\right)-\left(\nabla \cdot \boldsymbol{v}_{h}, p_{h}\right)-\left(\nabla \cdot \boldsymbol{v}_{h}, \tilde{p}\right)=\left\langle\boldsymbol{v}_{h}, \rho \boldsymbol{b}\right\rangle, \\
& \left(q_{h}, \nabla \cdot \boldsymbol{u}_{h}\right)+\left(q_{h}, \nabla \cdot \tilde{\boldsymbol{u}}\right)-\left(q_{h}, \frac{1}{\kappa} p_{h}\right)-\left(q_{h}, \frac{1}{\kappa} \tilde{p}\right)=0 .
\end{aligned}
$$

Here and in the following we have considered that $\overline{\boldsymbol{t}}=\mathbf{0}$ on $\Gamma_{N}$ for simplicity in the theoretical development, although arbitrary values for the tractions can be used with the proposed error estimator.

On the other hand, the equation for the subscales test functions in the element interiors are:

$$
\begin{aligned}
& \left(\boldsymbol{\epsilon}\left(\boldsymbol{v}^{\prime}\right), \boldsymbol{\sigma}^{\prime}\left(\boldsymbol{u}_{h}\right)\right)+\left(\boldsymbol{\epsilon}\left(\boldsymbol{v}^{\prime}\right), \boldsymbol{\sigma}^{\prime}(\tilde{\boldsymbol{u}})\right)-\left(\nabla \cdot \boldsymbol{v}^{\prime}, p_{h}\right)-\left(\nabla \cdot \boldsymbol{v}^{\prime}, \tilde{p}\right)=\left\langle\boldsymbol{v}^{\prime}, \rho \boldsymbol{b}\right\rangle, \\
& \left(q^{\prime}, \nabla \cdot \boldsymbol{u}_{h}\right)+\left(q^{\prime}, \nabla \cdot \tilde{\boldsymbol{u}}\right)-\left(q^{\prime}, \frac{1}{\kappa} p_{h}\right)-\left(q^{\prime}, \frac{1}{\kappa} \tilde{p}\right)=0 .
\end{aligned}
$$

The equations for the subscales cannot be exactly solved and need to be approximated. Integrating by parts equation (5) inside each element and neglecting the contribution of the pressure subscales on the displacement subscales equation we obtain the following equation:

$$
-\left(\boldsymbol{v}^{\prime}, \nabla \cdot\left(\boldsymbol{\sigma}^{\prime}\left(\boldsymbol{u}_{h}\right)\right)\right)_{h}-\left(\boldsymbol{v}^{\prime}, \nabla \cdot\left(\boldsymbol{\sigma}^{\prime}(\tilde{\boldsymbol{u}})\right)\right)_{h}+\left(\boldsymbol{v}^{\prime}, \nabla p_{h}\right)=\left\langle\boldsymbol{v}^{\prime}, \rho \boldsymbol{b}\right\rangle .
$$

In this expression, subscript $h$ is used to indicate that integrals are evaluated element-wise and we have taken into account that the exact fluxes (stresses) across inter-element boundaries cancel in matching element faces. It represents the equation for the subscales on the element interiors. Grouping terms for $\boldsymbol{u}_{h}$ and $\tilde{\boldsymbol{u}}$ we obtain:

$$
-\left(\boldsymbol{v}^{\prime}, \nabla \cdot\left(\boldsymbol{\sigma}^{\prime}(\tilde{\boldsymbol{u}})\right)\right)_{h}=\left(\boldsymbol{v}^{\prime}, \rho \boldsymbol{b}+\nabla \cdot \boldsymbol{\sigma}^{\prime}\left(\boldsymbol{u}_{h}\right)-\nabla p_{h}\right)_{h} .
$$


Finally, we introduce the additional key approximation

$$
\nabla \cdot\left(\boldsymbol{\sigma}^{\prime}(\tilde{\boldsymbol{u}})\right)=\tau^{-1} \tilde{\boldsymbol{u}}
$$

Assuming isotropic elasticity, the expression for $\tau$ in each element can be defined as (see [30]):

$$
\tau=c_{1} \frac{h^{2}}{2}\left(\frac{1}{\mu}+\frac{1}{\kappa}\right)
$$

with $h$ being the element size, $\mu$ being the shear modulus and $c_{1}$ being an algorithmic constant. A more elaborated expression for the stabilization parameter $\tau$ would be required if anisotropic materials are considered. For simplicity we have assumed quasi-uniform meshes, so that $\tau$ will be considered the same for all the elements in what follows.

The closed form expression for the subscales in the element interiors can now be obtained from equation (7):

$$
\tilde{\boldsymbol{u}}=\tau \tilde{P}\left(\rho \boldsymbol{b}+\nabla \cdot \boldsymbol{\sigma}^{\prime}\left(\boldsymbol{u}_{h}\right)-\nabla p_{h}\right) .
$$

where $\tilde{P}(\cdot)$ denotes the projection onto the subscales space. This projection operator is many times defined as the identity operator, which results in the approach known as the Algebraic Subgrid Scale (ASGS) method. In the case of the Orthogonal Subgrid Scale method (OSS) used in this work, the projection operator is taken as projection onto the space orthogonal to the finite element space:

$$
\tilde{P}=I-P_{h},
$$

where $P_{h}$ denotes the projection onto the appropriate finite element space, either of displacements as in (9) or onto the pressure space, as we require next.

Applying a similar strategy to equation (4) we arrive to the expression for the pressure subscales:

$$
\tilde{p}=\tau_{p} \tilde{P}\left(\frac{1}{\kappa} p_{h}-\nabla \cdot \boldsymbol{u}_{h}\right)=\tau_{p} \tilde{P}\left(-\nabla \cdot \boldsymbol{u}_{h}\right)
$$

with

$$
\tau_{p}=c_{2} 2\left(\frac{1}{\mu}+\frac{1}{\kappa}\right)^{-1} .
$$

We have assumed that the normal component of the stresses produced by the finite element solution and the subscales is continuous across interelement boundaries, but this does not happen when the subscales are approximated. We now argue as in [28] to obtain the contribution of the subscales on the element boundaries, assuming this does not affect the subscales in the element interiors. Integrating by parts within each element in equations (3) and (4), we obtain:

$$
\begin{gathered}
\left(\boldsymbol{\epsilon}\left(\boldsymbol{v}_{h}\right), \boldsymbol{\sigma}^{\prime}\left(\boldsymbol{u}_{h}\right)\right)-\left(\nabla \cdot \boldsymbol{\sigma}^{\prime}\left(\boldsymbol{v}_{h}\right), \tilde{\boldsymbol{u}}\right)_{h}+\sum_{K}\left\langle\boldsymbol{n} \cdot \boldsymbol{\sigma}^{\prime}\left(\boldsymbol{v}_{h}\right), \tilde{\boldsymbol{u}}_{E}\right\rangle_{\partial K} \\
-\left(\nabla \cdot \boldsymbol{v}_{h}, p_{h}\right)-\left(\nabla \cdot \boldsymbol{v}_{h}, \tilde{p}\right)=\left\langle\boldsymbol{v}_{h}, \rho \boldsymbol{b}\right\rangle, \\
\left(q_{h}, \nabla \cdot \boldsymbol{u}_{h}\right)-\left(\nabla q_{h}, \tilde{\boldsymbol{u}}\right)_{h}+\sum_{K}\left\langle q_{h}, \boldsymbol{n} \cdot \tilde{\boldsymbol{u}}_{E}\right\rangle_{\partial K}+\left(q_{h}, \frac{1}{\kappa} p_{h}\right)+\left(q_{h}, \frac{1}{\kappa} \tilde{p}\right)=0,
\end{gathered}
$$

where $\tilde{\boldsymbol{u}}_{E}$ represents the value for the subscales on the element boundaries $\partial K$. We assume it is uniquely defined on the edges (faces, in $3 \mathrm{D}$ ) shared by neighboring elements, that we denote with subscript $E$. The expression for the subscales in the element interiors has already been obtained in equations (9) and (10). However, their contribution in the element boundaries 
$\tilde{\boldsymbol{u}}_{E}$ in equations (11) and (12) still needs to be accounted for. Following [28], the subscales in the element boundaries can be approximated as:

$$
\tilde{\boldsymbol{u}}_{E}=-\tau_{E} \llbracket \boldsymbol{\sigma}^{\prime}\left(\boldsymbol{u}_{h}\right) \cdot \boldsymbol{n}-p_{h} \boldsymbol{n} \rrbracket_{E},
$$

with

$$
\tau_{E}=\frac{\delta_{0} h}{2 \mu} .
$$

The algorithmic constant $\delta_{0}=1 / 2$ is typically used. $h$ here is taken as the average element length, that is, the average of the square (in two dimensions) or cubic (in three dimensions) root of the volume of the elements owning the edge or face. The jump operator across a face $E$ is defined as:

$$
\llbracket \boldsymbol{n} g \rrbracket_{E}=\left.\boldsymbol{n}_{1} g\right|_{\partial K_{1} \cap E}+\left.\boldsymbol{n}_{2} g\right|_{\partial K_{2} \cap E},
$$

where $\boldsymbol{n}_{i}$ denotes the external normal in the boundary of element $K_{i}$. This expression for the boundary subscales is obtained by enforcing that the total tractions are continuous across interelement boundaries. As a consequence, $\llbracket \boldsymbol{\sigma}^{\prime}(\boldsymbol{u}) \cdot \boldsymbol{n}-p \boldsymbol{n} \rrbracket=0$ must hold. Decomposing this expression into the finite element contribution and the contribution of the displacement boundary subscales we obtain:

$$
\llbracket \boldsymbol{\sigma}^{\prime}\left(\boldsymbol{u}_{h}+\tilde{\boldsymbol{u}}_{E}\right) \cdot \boldsymbol{n}-p \boldsymbol{n} \rrbracket=0
$$

Finally, approximating $\llbracket \boldsymbol{\sigma}^{\prime}\left(\tilde{\boldsymbol{u}}_{E}\right) \cdot \boldsymbol{n} \rrbracket$ on the faces of the elements as:

$$
\llbracket \boldsymbol{\sigma}^{\prime}\left(\tilde{\boldsymbol{u}}_{E}\right) \cdot \boldsymbol{n} \rrbracket=\frac{\tilde{\boldsymbol{u}}_{E}}{\tau_{E}}
$$

yields (13). The details of the formulation can be found in [28]. A similar model for the boundary subscales in the context of the transport equation is proposed in [16].

For continuous interpolation spaces for $p_{h}$, where the pressure is already continuous, we obtain:

$$
\tilde{\boldsymbol{u}}_{E}=-\tau_{E} \llbracket \boldsymbol{\sigma}^{\prime}\left(\boldsymbol{u}_{h}\right) \cdot \boldsymbol{n} \rrbracket_{E} .
$$

Introducing the previous expressions for the displacement and pressure subscales in equations (3) and (4) results in a stabilized formulation capable of dealing with any interpolation space pair for the displacement and pressure, even in the incompressible limit. However, when using continuous interpolation spaces for both the displacement and the pressure, not all of the previous terms are required in order to obtain a stable formulation. Particularly, a stable formulation can be obtained by dropping all the contributions of the subgrid scale terms except for the ones corresponding to the element interior displacement subscales in the continuity equation, $\sum_{K} \tau\left\langle\nabla q_{h}, \tilde{P}\left(\rho \boldsymbol{b}+\nabla \cdot \boldsymbol{\sigma}^{\prime}\left(\boldsymbol{u}_{h}\right)-\nabla p_{h}\right)\right\rangle_{K}$. When using continuous interpolation spaces this results in the simplest implementation without harming the proper rate of convergence (see [27]). The additional terms can enhance the solution and account for the effect of unresolved subscales and materials with complex behavior (see for instance $[31,28,32])$.

Due to this, and in order to obtain an as simple to implement as possible formulation without harming the accuracy of the method, we are going to use an OSS bilinear form with only the indispensable stabilizing terms in order to build the finite element system of equations. On the contrary, the full approximation for the subscales is going to be used in order to compute an as accurate as possible error estimator in Section 3.

Taking this into account, the final stabilized weak form of the problem is:

$$
B_{S}\left(\left[\boldsymbol{u}_{h}, p_{h}\right],\left[\boldsymbol{v}_{h}, q_{h}\right]\right)=L_{S}\left(\boldsymbol{v}_{h}, q_{h}\right)
$$


with

$$
\begin{aligned}
B_{S}\left(\left[\boldsymbol{u}_{h}, p_{h}\right],\left[\boldsymbol{v}_{h}, q_{h}\right]\right) & =B\left(\left[\boldsymbol{u}_{h}, p_{h}\right],\left[\boldsymbol{v}_{h}, q_{h}\right]\right)+\sum_{K} \tau\left\langle\nabla q_{h}, \tilde{P}\left(-\nabla \cdot \boldsymbol{\sigma}^{\prime}\left(\boldsymbol{u}_{h}\right)+\nabla p_{h}\right)\right\rangle_{K} \\
L_{S}\left(\boldsymbol{v}_{h}, q_{h}\right) & =L\left(\boldsymbol{v}_{h}, q_{h}\right)+\sum_{K} \tau\left\langle\nabla q_{h}, \tilde{P}(\rho \boldsymbol{b})\right\rangle_{K}
\end{aligned}
$$

The main properties of the formulation are the following: first, the formulation can now be shown to be stable even in the incompressible limit (see [33, 34] for a stability proof), and any set of continuous interpolation spaces $V_{h}$ and $Q_{h}$ can be used. Second, the stabilization term is consistent. As a consequence, the convergence rate of the error of the finite element solution with respect to the mesh size is not harmed by the stabilizing term.

Remark 1. When implementing the OSS formulation, the projections onto the finite element space, $P_{h}$, need to be computed. A possible approach to deal with these projections is to compute them in an iterative manner. This means that the global $L^{2}$ projections onto the finite element space are evaluated using the residual fields at the preceding iteration. For non-linear problems the iterative process for the orthogonal projection can be coupled with the non-linear iterations, generally with reduced impact in the convergence rate.

For linear problems, an iterative loop (not required in the original problem) is necessary to compute the projections. Local projections can be used to avoid this issue, although we have not pursued this possibility in this work, because our final objective is to apply the proposed methodology to non-linear solid mechanics problems.

Also, diagonal lumped mass matrices can be used to perform low-computational-cost $L^{2}$ projections, with low impact in the accuracy of the results. This is the strategy followed in the numerical examples section.

Remark 2. Note that, following [28], the expression for the subscales on the element boundaries is the same for our definition of the ASGS and the OSS method, that is, an identity projection operator is used for the subscales on the element boundaries. An unexplored possibility would be to consider an edge or face orthogonal projection for the subscales on the element boundaries. However, it is not clear that this would lead to a sharper error estimator.

\section{Variational Multiscale based error estimators}

\subsection{Definition of the error estimator}

In this work we are going to see that the norm of the orthogonal subgrid scales given by equations (9), (10) and (13) can be used as an error estimator and provide an accurate measure of the difference between the finite element solution and the exact one. For this, we consider as an error estimator the following norm of the subscales:

$$
\eta_{K}^{2}:=\tau^{-1} \int_{K}|\tilde{\boldsymbol{u}}|^{2}+\tau_{p}^{-1} \int_{K}|\tilde{p}|^{2}+\tau_{E}^{-1} \int_{\partial K}\left|\tilde{\boldsymbol{u}}_{E}\right|^{2}
$$

Following [35] as done in [4], the last term in (15) can be shown to be equivalent to $\| \boldsymbol{\sigma}_{h}^{\prime}-$ $P_{h}\left(\sigma_{h}^{\prime}\right) \|_{K}^{2}$ in the sense that:

$$
\gamma_{1} \sum_{K} h_{K} \int_{\partial K}\left|\llbracket \boldsymbol{\sigma}^{\prime}\left(\boldsymbol{u}_{h}\right) \cdot \boldsymbol{n} \rrbracket_{E}\right|^{2} \leq\left\|\boldsymbol{\sigma}_{h}^{\prime}-P_{h}\left(\boldsymbol{\sigma}_{h}^{\prime}\right)\right\|^{2} \leq \gamma_{2} \sum_{K} h_{K} \int_{\partial K}\left|\llbracket \boldsymbol{\sigma}^{\prime}\left(\boldsymbol{u}_{h}\right) \cdot \boldsymbol{n} \rrbracket_{E}\right|^{2}
$$

for certain positive constants $\gamma_{1}, \gamma_{2}$. This introduces the possibility of approximating:

$$
\tau_{E}^{-1} \int_{\partial K}\left|\tilde{\boldsymbol{u}}_{E}\right|^{2} \approx \tau_{E} \gamma h_{K}^{-1}\left\|\tilde{P}\left(\boldsymbol{\sigma}_{h}^{\prime}\right)\right\|_{K}^{2}
$$


in practical cases. The most convenient $\gamma$ parameter can be obtained through a numerical benchmark. In the numerical examples in Section 4 this approximation has been used. The performance of the error estimator (15) is going to be compared with the measure of the actual error in the energy norm, which we define as follows:

$$
\|[\boldsymbol{u}, p]\|^{2}:=\left(\boldsymbol{\sigma}^{\prime}(\boldsymbol{u}), \boldsymbol{\epsilon}(\boldsymbol{u})\right)+\frac{1}{\kappa}\|p\|^{2}+\sum_{K} \tau\|\nabla p\|_{K}^{2} .
$$

This is the norm in which stability and convergence can be proved for the stabilized finite element formulation being used.

\subsection{Error estimator bound and approximation}

In the following we check that the orthogonal subgrid scales based error estimator effectively provides an upper bound for the energy norm error. We define the error and the error's energy norm as:

$$
\begin{gathered}
e=\left[\boldsymbol{u}-\boldsymbol{u}_{h}, p-p_{h}\right], \\
e=\left\|\left[\boldsymbol{u}-\boldsymbol{u}_{h}, p-p_{h}\right]\right\| .
\end{gathered}
$$

In order to show that the orthogonal subgrid scales can be used as an error estimator, we will show that the following inequality holds:

$$
\|\boldsymbol{e}\|^{2} \leq C_{\mathrm{EST}}^{2}\left(\sum_{K} \eta_{K}^{2}\right),
$$

where $C_{\mathrm{EST}}$ is a mesh geometry dependent constant. This can be obtained following the procedure explained in [36]. The only particularity is that, since we consider the subscales to belong to spaces orthogonal to the finite element interpolation, some simplifications can be done in the derivation process. In particular, it can be shown that the upper bound for the error estimator is sharper than in the case of standard (non orthogonal) approximation of the subscales: basically the same error estimator as in the ASGS method can be obtained for the OSS method, but using the orthogonal projection of the residual. Since the norm of the orthogonal projection of the residual is smaller than the norm of the residual, the upper bound of the error estimator is sharper.

Due to the consistency of the stability term, the usual Galerkin orthogonality for the stabilized formulation (14) can be applied:

$$
B_{S}\left(\boldsymbol{e},\left[\boldsymbol{v}_{h}, q_{h}\right]\right)=0 \quad \forall \boldsymbol{v}_{h} \in V_{h}, \forall q_{h} \in Q_{h} .
$$

Also:

$$
\begin{aligned}
B_{S}(\boldsymbol{e},[\boldsymbol{v}, q]) & =B_{S}([\boldsymbol{u}, p],[\boldsymbol{v}, q])-B_{S}\left(\left[\boldsymbol{u}_{h}, p_{h}\right],[\boldsymbol{v}, q]\right) \\
& =L_{S}(\boldsymbol{v}, q)-B_{S}\left(\left[\boldsymbol{u}_{h}, p_{h}\right],[\boldsymbol{v}, q]\right) .
\end{aligned}
$$

Now, departing from the originally stabilized weak form (14), separating integrals within each element and integrating by parts we arrive to:

$$
\begin{aligned}
B_{S}(\boldsymbol{e},[\boldsymbol{v}, q]) & =\sum_{K}\left\langle\boldsymbol{v}, \rho \boldsymbol{b}+\nabla \cdot \boldsymbol{\sigma}^{\prime}\left(\boldsymbol{u}_{h}\right)-\nabla p_{h}\right\rangle_{K} \\
& -\sum_{K}\left\langle\boldsymbol{v}, \boldsymbol{n} \cdot\left(\boldsymbol{\sigma}^{\prime}\left(\boldsymbol{u}_{h}\right)-p_{h} \boldsymbol{I}\right)\right\rangle_{\partial K}-\sum_{K}\left\langle q, \nabla \cdot \boldsymbol{u}_{h}+\frac{1}{\kappa} p_{h}\right\rangle_{K} \\
& +\sum_{K} \tau\left\langle\nabla q, \tilde{P}\left(\rho \boldsymbol{b}+\nabla \cdot \boldsymbol{\sigma}^{\prime}\left(\boldsymbol{u}_{h}\right)-\nabla p_{h}\right)\right\rangle_{K} .
\end{aligned}
$$


Making use of (18), we have:

$$
B_{S}(\boldsymbol{e},[\boldsymbol{v}, q])=B_{S}\left(\boldsymbol{e},\left[\boldsymbol{v}-\boldsymbol{v}_{h}, q-q_{h}\right]\right) .
$$

Now, in the previous equation we take $\boldsymbol{v}_{h}=P_{h}(\boldsymbol{v}), q_{h}=P_{h}(q)$ and we recall that $\boldsymbol{v}-P_{h}(\boldsymbol{v})=$ $\tilde{P}(\boldsymbol{v})$ for the OSS method:

$$
\begin{aligned}
B_{S}(\boldsymbol{e},[\boldsymbol{v}, q]) & =\sum_{K}\left\langle\tilde{P}(\boldsymbol{v}), \tilde{P}\left(\rho \boldsymbol{b}+\nabla \cdot \boldsymbol{\sigma}^{\prime}\left(\boldsymbol{u}_{h}\right)-\nabla p_{h}\right)\right\rangle_{K} \\
& -\sum_{E}\left\langle\tilde{P}(\boldsymbol{v}), \llbracket \boldsymbol{n} \cdot \boldsymbol{\sigma}^{\prime}\left(\boldsymbol{u}_{h}\right)-p_{h} \boldsymbol{n} \rrbracket\right\rangle_{E}-\sum_{K}\left\langle\tilde{P}(q), \tilde{P}\left(\nabla \cdot \boldsymbol{u}_{h}\right)\right\rangle_{K} \\
& +\sum_{K} \tau\left\langle\tilde{P}(\nabla(\tilde{P}(q))), \tilde{P}\left(\rho \boldsymbol{b}+\nabla \cdot \boldsymbol{\sigma}^{\prime}\left(\boldsymbol{u}_{h}\right)-\nabla p_{h}\right)\right\rangle_{K} .
\end{aligned}
$$

Due to interpolation properties of the finite element space (see for instance [37]) and the definition of $\tilde{P}$, the following inequalities hold:

$$
\begin{aligned}
& \sum_{K}\|\tilde{P}(\boldsymbol{v})\|_{K} \lesssim \tau^{1 / 2}\|[\boldsymbol{v}, 0]\| \\
& \sum_{K}\|\tilde{P}(\boldsymbol{v})\|_{\partial K} \lesssim \tau_{E}^{1 / 2}\|[\boldsymbol{v}, 0]\| \\
& \sum_{K}\|\tilde{P}(q)\|_{K} \lesssim \tau_{p}^{1 / 2}\|\|[0, q] \| \\
& \sum_{K} \tau^{1 / 2}\|\tilde{P}(\nabla(\tilde{P}(q)))\|_{K} \lesssim \|[[0, q] \|
\end{aligned}
$$

where $\lesssim, \gtrsim$ denote an inequality up to dimensionless constants independent of the discretization.

Using the stability of $B_{S}$ and applying the triangular inequality we can say that there exists $[\boldsymbol{v}, q]$ such that:

$$
\|\boldsymbol{e}\|\|\|[\boldsymbol{v}, q] \| \mid \lesssim B_{S}(\boldsymbol{e},[\boldsymbol{v}, q]) .
$$

The proof for the stability of $B_{S}$ can be found in [33,34], and has not been included for conciseness. Let us only remark that in order for $B_{S}$ to be stable, a weakened inf-sup compatibility condition needs to be satisfied between $V_{h}$ and $Q_{h}$. This weakened inf-sup condition is satisfied by most $\boldsymbol{u}-p$ interpolation pairs, and in particular by equal order interpolation spaces for $\boldsymbol{u}$ and $p$. Now, taking $[\boldsymbol{v}, q]$ such that (20) is fulfilled and using Schwarz's inequality in (19), the sought bound for the error is obtained:

$$
\|\boldsymbol{e}\|^{2} \lesssim \sum_{K} \eta_{K}^{2}
$$

It is interesting to note that the obtained error bound is sharper if we use an orthogonal space for the subgrid scales (OSS method), than if the usual space for the subscales is used (ASGS). This observation leads to our error estimator based on the OSS method.

\section{$4 \quad$ Numerical examples}

In this section some numerical examples are presented which focus in the various features of the OSS error estimator. Firstly, some analytical examples that highlight the behavior of the estimator with different type of displacement and pressure solution fields are simulated. 
This allows us to see how does the estimator behave in divergence free, compressible and incompressible, and hydrostatic solution fields. In each of these examples, we evaluate the weight of the contribution of the interior and boundary subscales, and we compare the error estimator obtained with the OSS and the ASGS methods.

A second block of examples show the behavior of the error estimator when applied to problems where non-linear constitutive models are used.

\subsection{Divergence free solution for a compressible material}

In this numerical example the following analytical solution is tested:

$$
u=2 x y, \quad v=-y^{2},
$$

where $u$ and $v$ are the Cartesian components of the displacement. The computational domain is $\Omega:=(0,1) \times(0,1)$. A mesh of linear triangles is used to discretize the computational domain. The Poisson coefficient is taken as $\nu=0.21$, which corresponds to a compressible material. The Young modulus is taken as $E=2.40 \cdot 10^{11}$, which results in a bulk modulus $\kappa=1.3793 \cdot 10^{11}$ and a shear modulus $\mu=9.9174 \cdot 10^{10}$. On the other hand, note that the analytical solution is divergence free, so the pressure is going to be zero everywhere in the domain. This solution is very convenient in order to analyze the contribution of the subscales on the element boundaries $\tilde{\boldsymbol{u}}_{E}$ to the error estimator, since the contributions of $\tilde{\boldsymbol{u}}$ and $\tilde{p}$ will be negligible. The subscales based error estimator is compared with the energy norm error. Since this is a smooth solution, the error estimator causes the algorithm to refine the mesh uniformly. Also, it is important to note that this example has been used to adjust the $\gamma$ parameter in approximation (17). We use $\gamma=4$ in all the numerical examples. Nevertheless, the spatial distribution of the error estimator is independent of the chosen $\gamma$ constant and it can be seen how it properly matches the spatial distribution of the energy norm error.

The energy norm error and the error estimated by using the norm of the subgrid scales are shown in Fig. 1, after some refinement steps have been done. It is interesting to observe that the shape of the elements that conformed the original mesh (which has been successively refined) can still be appreciated in the coloring of both the energy norm and the subscales error estimator. The cause for this is that the original coarse element geometry and quality determine the quality of their children refined elements. Fig. 2 shows the convergence rate of both the energy error and the subscales error estimator with the mesh size. It also shows the global efficiency index of the error estimator for this problem. It can be observed that the efficiency of the estimator for this case is very good.

\subsection{Hydrostatic solution for an incompressible material}

In this numerical example, an incompressible material with Poisson coefficient $\nu=0.5$ is tested, so that the bulk modulus is $\kappa=\infty$. The Young modulus is again taken as $E=$ $2.40 \cdot 10^{11}$ and a shear modulus $\mu=8 \cdot 10^{10}$. In this case an analytical solution with null displacement is considered:

$$
u=0, \quad v=0, \quad p=2 x^{2} y .
$$

The domain is again $\Omega:=(0,1) \times(0,1)$. A mesh of linear triangles is used to discretize the computational domain. As in the previous case, the solution is quite smooth, so a uniform refinement criteria is obtained. The main interest of this case is that both the contribution from the subscales on the element boundaries $\tilde{\boldsymbol{u}}_{E}$ and the contribution of the pressure subscales $\tilde{p}$ to the error indicator are negligible, so it allows us to evaluate the 

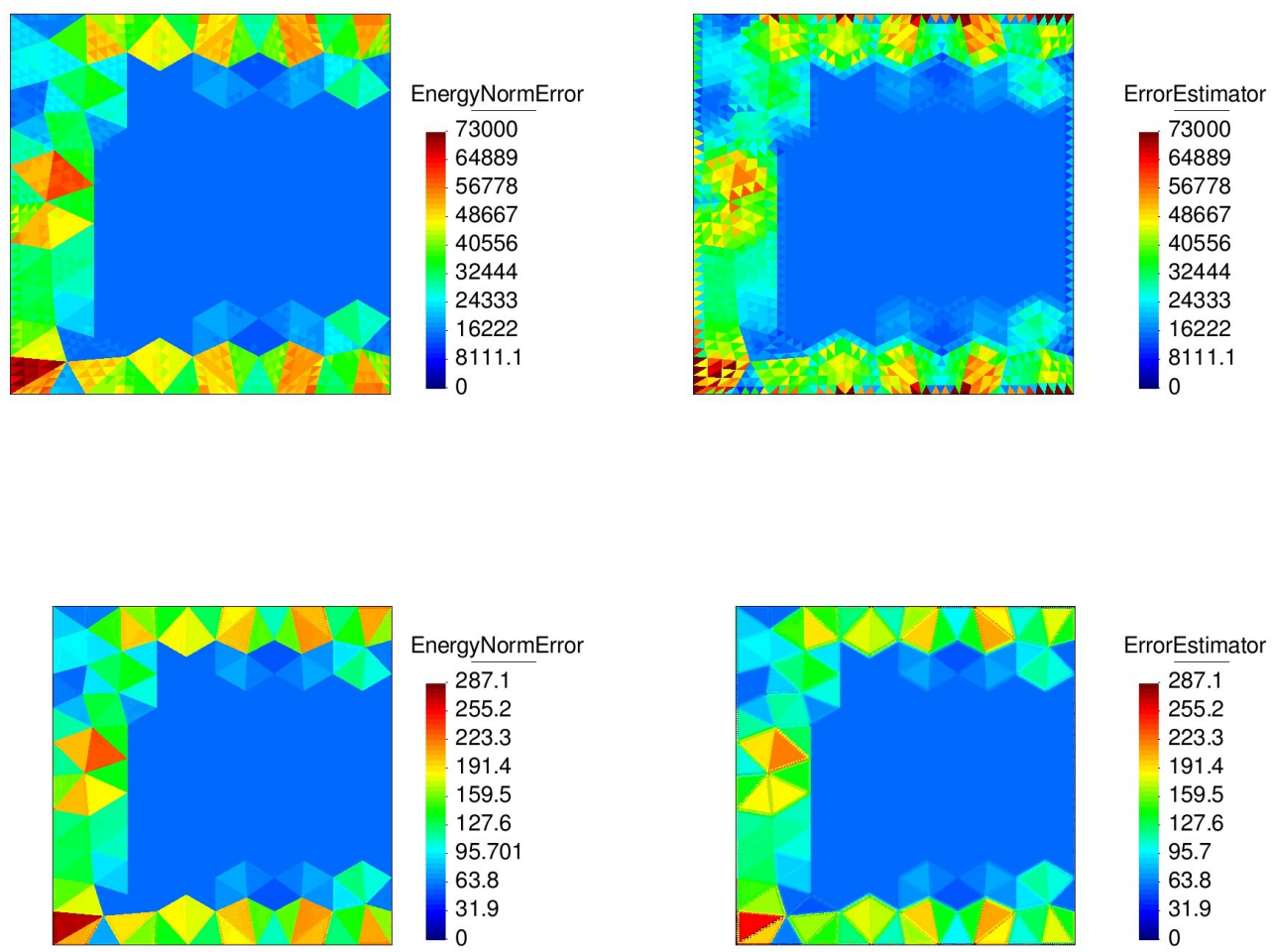

Figure 1: Left: Energy norm error contribution. Right: Subscales error estimator contribution. Top: After two refinement steps. Bottom: After four refinement steps.
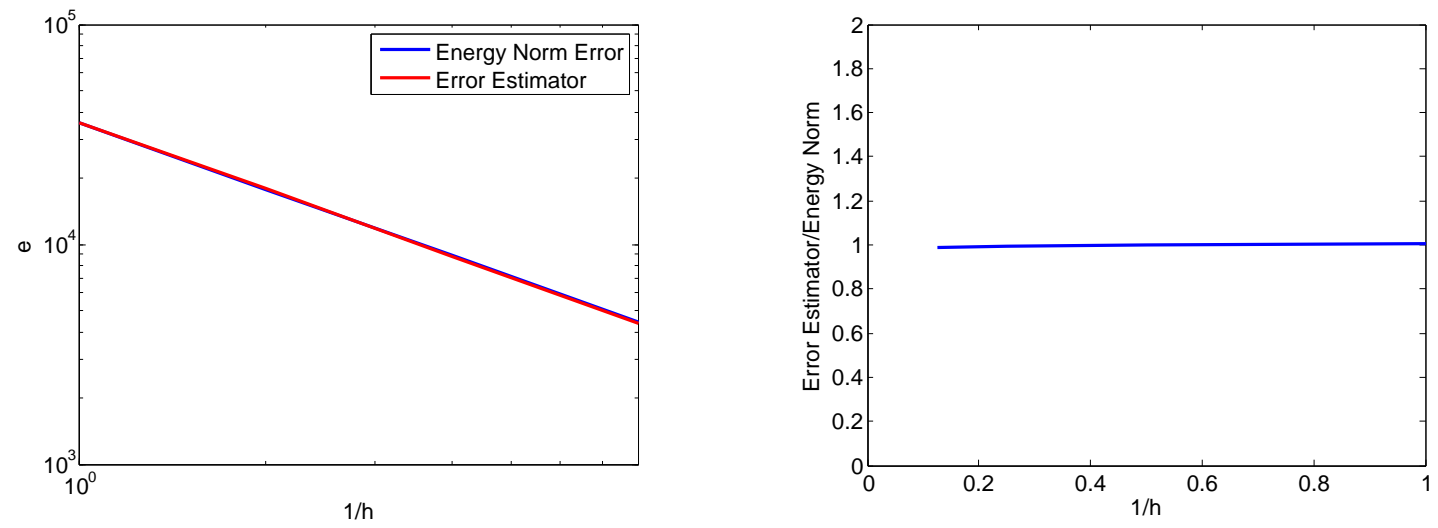

Figure 2: Left: convergence of the energy error norm and the subscales error estimator with the mesh size. Right: Global efficiency index as a function of the mesh size. 

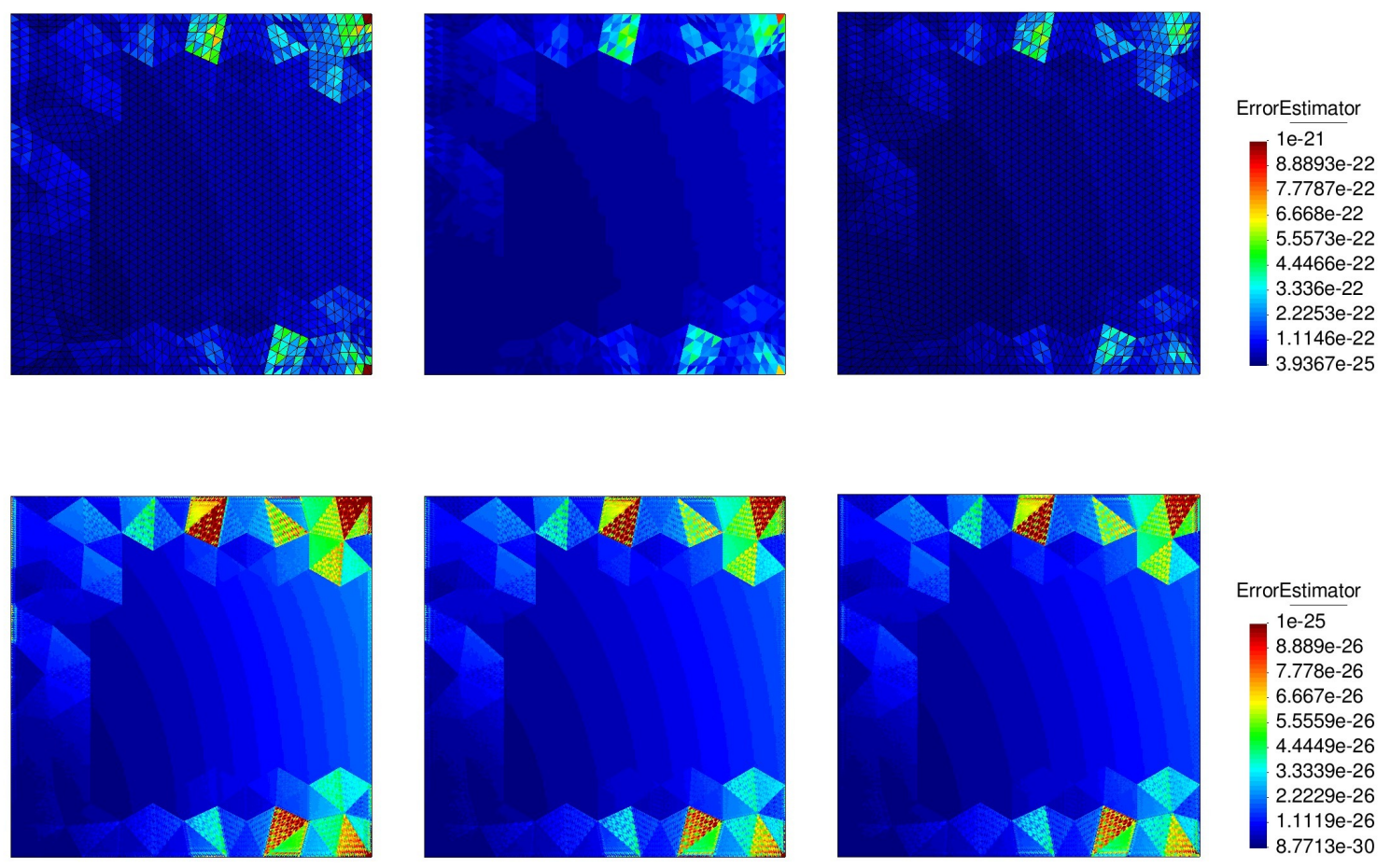

Figure 3: Left: Energy Error Norm. Center: ASGS error estimator. Right: OSS error estimator. Top: Two refinement levels. Bottom: Four refinement levels.

performance of $\tilde{\boldsymbol{u}}$ as an error indicator. Fig. 3 shows a comparison between the energy norm error, the Algebraic Subgid Scale (ASGS) error estimator (obtained by using the identity projector $I$ instead of $\tilde{P}$ when defining the subgrid scales in (9)) and the OSS error estimator. The ASGS is the usual approach in VMS methodologies, possibly because it allows to avoid the projection onto the subscales space. However, it has been observed that in many cases the OSS approach results in more accurate results (see [31, 38]).

It can be observed that a good match between the energy norm error and the ASGS and OSS error estimators is obtained. Despite the fact that the analysis shows that the OSS error bound is sharper than the ASGS error bound, in this practical example very few differences are observed between them. In Fig. 4 a comparison between the convergence rate of the energy norm error and the OSS error estimator is shown, together with the global efficiency index of the OSS error estimator. It can be observed that the error estimator does a good job in reproducing the convergence rate of the energy norm error.

\subsection{Hydrostatic solution for a compressible material}

This case deals with a hydrostatic solution for a compressible material. The contribution of the pressure gradient to the error and the capability of the error estimator to capture it are 

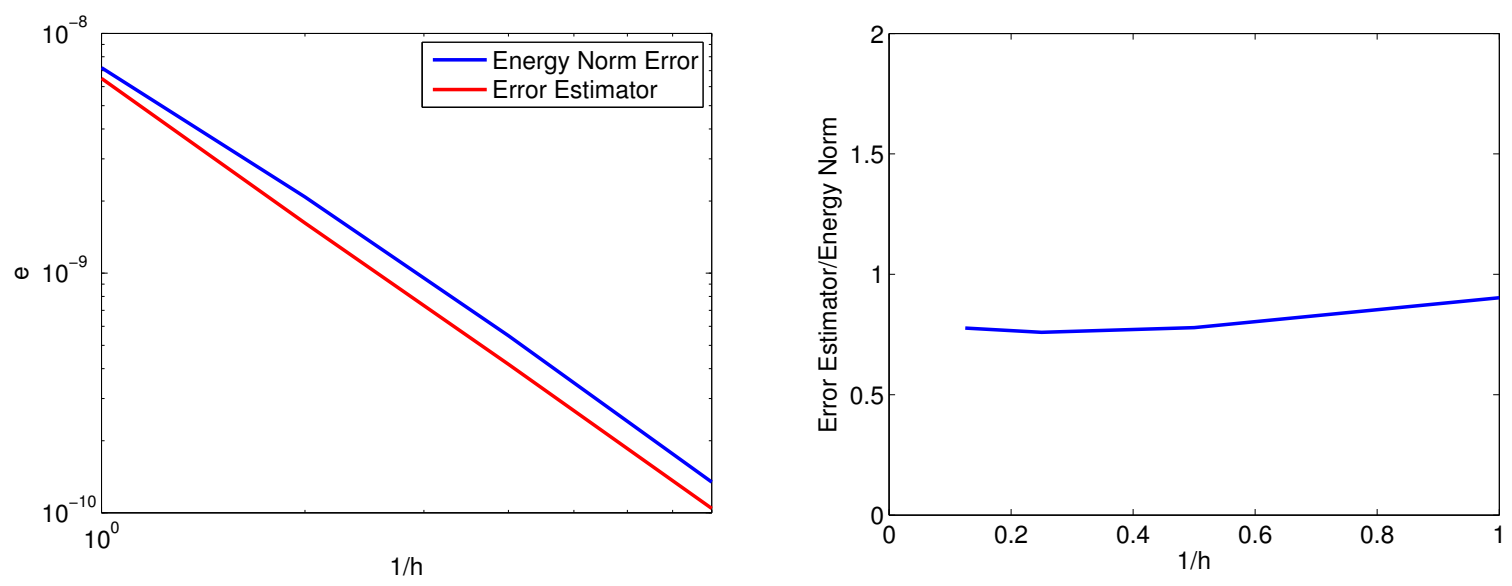

Figure 4: Convergence rate and global efficiency index for the error estimator.

evaluated. The components of the displacement of the considered solution are:

$$
\begin{aligned}
u & =\frac{1}{2}\left(x^{2}-y^{2}-z^{2}\right)+x(y+z)+y+z, \\
v & =\frac{1}{2}\left(y^{2}-x^{2}-z^{2}\right)+y(x+z)-z-x, \\
w & =\frac{1}{2}\left(z^{2}-x^{2}-y^{2}\right)+z(x+y)-x+y .
\end{aligned}
$$

The Poisson coefficient is taken as $\nu=0$, and the Young modulus $E=1$, which results in a bulk modulus $\kappa=1 / 3$ and a shear modulus $\mu=0.5$. In this case the computational domain is three-dimensional and occupies the cube $\Omega:=(0,1) \times(0,1) \times(0,1)$. A mesh of linear tetrahedra is used to discretize the computational domain. The resulting stress field is hydrostatic, the value for the pressure being:

$$
p=-\kappa(3 x+3 y+3 z) .
$$

As in the previous cases, we are using linear approximation spaces for the displacement and pressure unknowns. As a consequence, the pressure approximation is capable of exactly capturing the analytical solution. However, the displacement approximation does not allow one to exactly represent the displacement field and its divergence, which results in an approximation error. Since the stresses are purely hydrostatic, the only active part of the error estimator will be the pressure subscale $\tilde{p}$, which includes the orthogonal projection of the displacement divergence. This is precisely the part of the error estimator that we want to evaluate in this numerical example. In this case, no difference will be appreciated between using the algebraic or the orthogonal subscales version for the error estimator, because when using equal order interpolation the pressure is precisely the projection onto the finite element space of the displacement divergence, and the expression for the pressure subscale is the same for the ASGS and the OSS error estimators.

Fig. 5 shows a comparison of the energy norm error distribution and the error estimator at several refinement steps. Both are similar at the several stages, and thus we conclude that the pressure subscales are capable of capturing the part of the error associated to hydrostatic stress states.

Fig. 6 shows the convergence and global efficiency index for the hydrostatic solution for a compressible material. It can be observed that the global efficiency index is close to one in the whole refinement process and that the energy norm error and the global efficiency index converge at the same rate. 

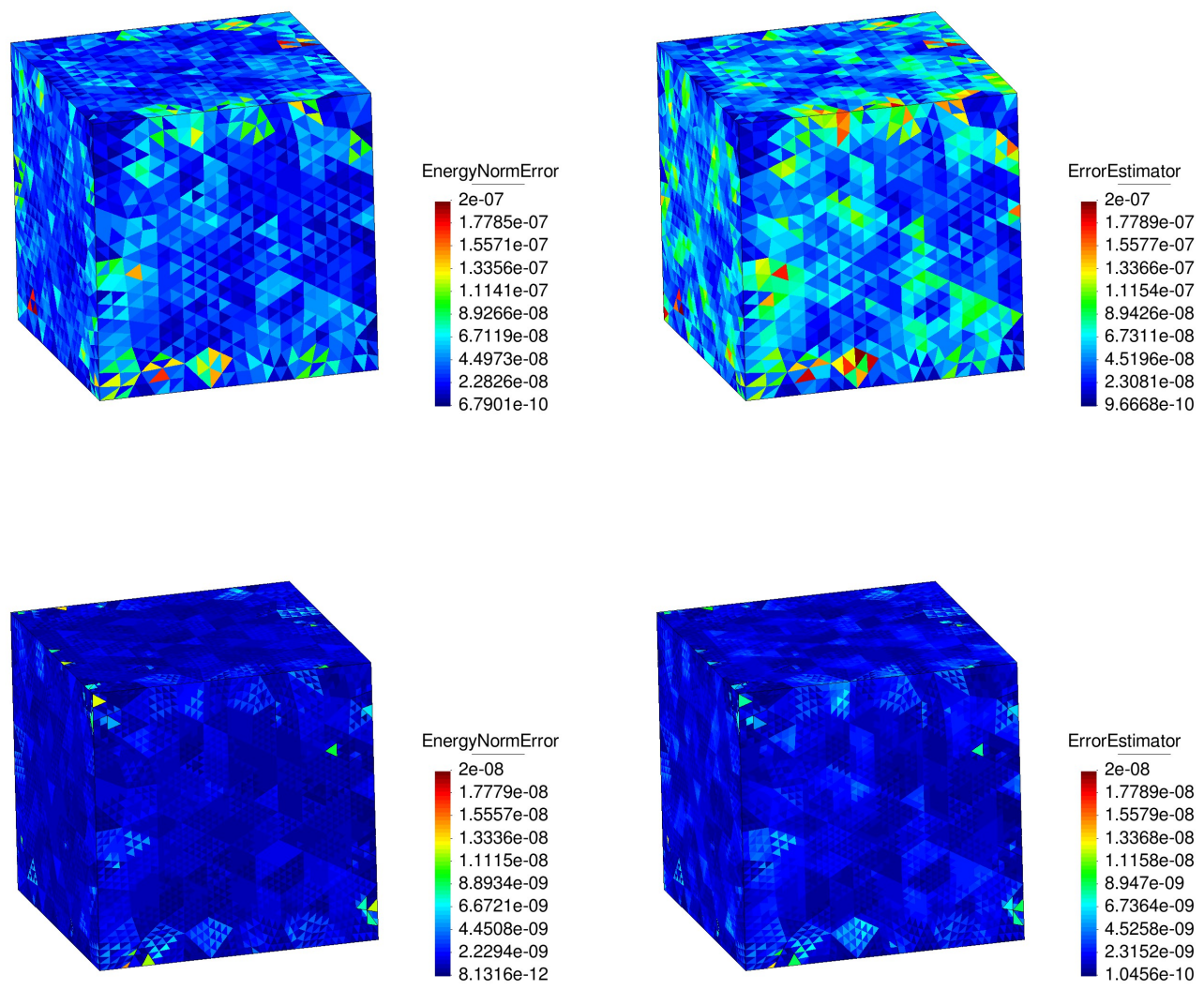

Figure 5: Comparison of the energy norm error and the subgrid scales error estimator for the hydrostatic solution for a compressible material. The figures in the left represent the energy norm error, the ones in the right the subgrid scales error estimator. From top to bottom, the results for one and two refinement steps are shown.
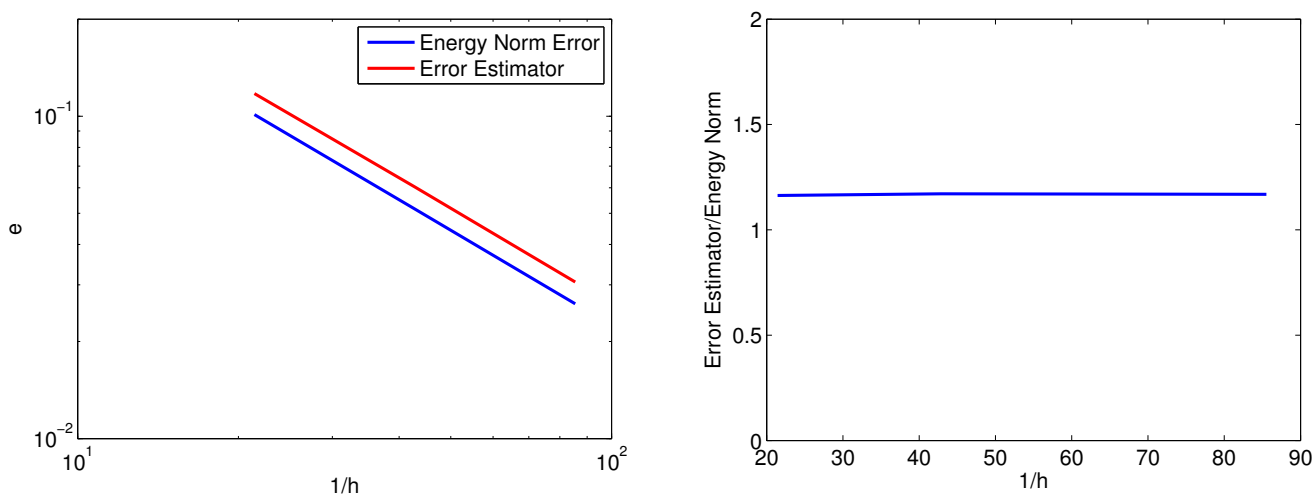

Figure 6: Left: Error and error estimator convergence for the hydrostatic solution for a compressible material. Right: Global efficiency index for the hydrostatic solution for a compressible material. 

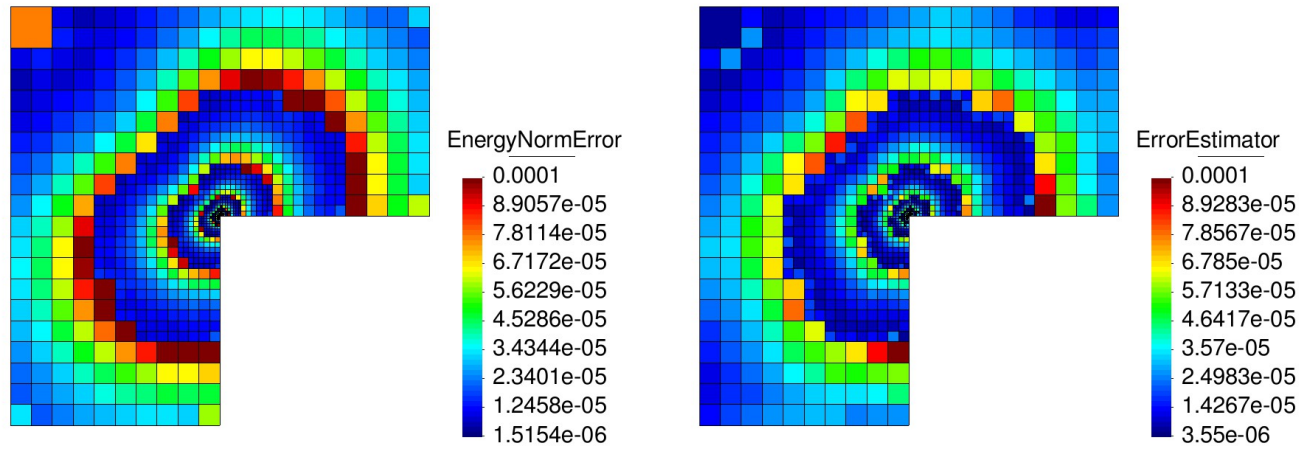

Figure 7: Comparison of the energy norm error and the orthogonal subgrid scales error estimator for the singular solution problem.

\subsection{Singular solution}

In this numerical example we test a solution for the incompressible limit $\nu=0.5$ (and thus $\kappa=\infty$ ) with a singular point. The Young modulus is taken as $E=1$, which results in a shear modulus $\mu=1 / 3$. This allows us to evaluate the performance of the subscales as an error indicator when large localized gradients appear. The studied solution is the following:

$\boldsymbol{u}(r, \phi)=r^{\alpha}\left[\begin{array}{c}\cos (\phi) \psi^{\prime}(\phi)+(1+\alpha) \sin (\phi) \psi(\phi) \\ \sin (\phi) \psi^{\prime}(\phi)-(1+\alpha) \sin (\phi) \psi(\phi)\end{array}\right], \quad p(r, \phi)=-r^{\alpha-1} \frac{(1+\alpha)^{2} \psi^{\prime}(\phi)+\psi^{\prime \prime \prime}(\phi)}{1-\alpha}$,

with $r$ and $\phi$ being the polar coordinates and:

$$
\psi(\phi)=\frac{\sin ((1+\alpha) \phi) \cos (\alpha \omega)}{1+\alpha}-\cos ((1+\alpha) \phi)+\frac{\sin ((\alpha-1) \phi) \cos (\alpha \omega)}{1-\alpha}+\cos ((\alpha-1) \phi) .
$$

Here we take $\omega=3 \pi / 2$ and $\alpha$ as the (approximate) root of the following non-linear equation:

$$
\frac{\sin ^{2}(\alpha \omega)-\alpha^{2} \sin ^{2}(\omega)}{\alpha^{2}}=0
$$

This solution is evaluated in the L-shaped domain:

$$
\Omega:=((-1,1) \times(-1,1)) \backslash([0,1] \times[-1,0]) .
$$

A bilinear quadrilateral mesh is used to discretize the computational domain.

Fig. 7 shows the comparison of the error estimator and the energy norm error after several refinement steps. It can be observed that both quantities are very similar, from which we conclude that the orthogonal subgrid scale error estimator is suitable also for problems in which the solution presents large gradients. Fig. 8 shows the convergence rate of the energy norm error compared to the error estimator and the global efficiency index. Again, very good results are obtained.

\subsection{Damage model}

In this numerical example we use the subscales error estimator in order to track the failure process of a plain strain test plate subject to an increasing axial effort. In order to properly track the evolution of the failure lines when using low order elements, a smoothing of the 

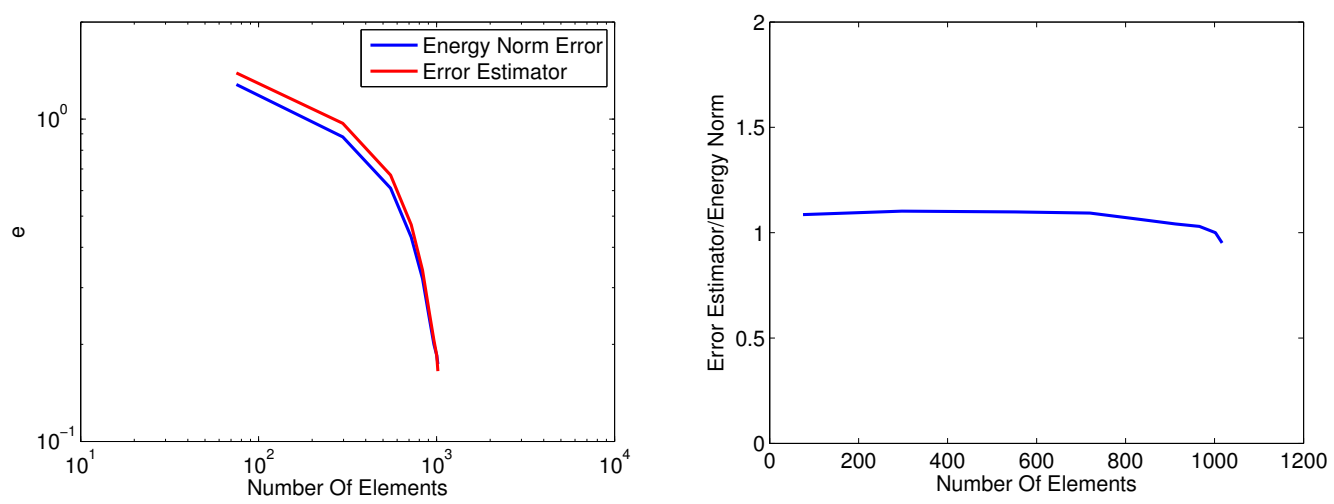

Figure 8: Convergence rate and global efficiency index for the singular solution case.

stress field is used in order to evaluate the damage constitutive model. This allows us to obtain an accuracy similar to the one of mixed $\boldsymbol{\sigma}$ - $\boldsymbol{u}$ - $p$ finite element formulations for solid mechanics, but without the additional complexity of solving a coupled stress field.

The constitutive equation for the J2-damage model used in this example (see [39]) reads as follows: an internal damage variable $d$ is introduced which takes into account the damage of the deviatoric part of the stresses. This leads to the modified constitutive equation:

$$
\boldsymbol{\sigma}=-p \boldsymbol{I}+(1-d) \mathcal{C}^{\prime}: \boldsymbol{\epsilon}(\boldsymbol{u}) .
$$

The characterization of damage is done through an equivalent one dimensional shear stress:

$$
\tau_{d}=\sqrt{\frac{3}{2} \boldsymbol{\sigma}^{\prime}: \boldsymbol{\sigma}^{\prime}},
$$

which corresponds to the classical Von Misses stress. We also define the explicit internal variable $r$ as:

$$
r=\max \left\{r_{0}, \max \left(\tau_{d}\right)\right\},
$$

where $r_{0}$ corresponds to the initial damage uniaxial stress. From here, the damage parameter can be computed as:

$$
d(r)=1-\frac{r_{0}}{r} \exp \left(-2 H_{S}\left(\frac{r-r_{0}}{r_{0}}\right)\right),
$$

where $H_{s}$ is a constant which depends on the considered material rate of energy dissipation, with:

$$
\begin{aligned}
H_{S} & =\frac{\bar{H}_{S} l_{c h}}{1-\bar{H}_{S} l_{c h}} \geq 0, \\
\bar{H}_{S} & =\frac{r_{0}^{2}}{3(2 \mu) \mathcal{G}_{I I}},
\end{aligned}
$$

where $l_{c h}$ is the fracture length (typically taken as the finite element size) and $\mathcal{G}_{I I}$ is the mode II fracture energy per unit area.

The considered problem is the following: a rectangular plate with an orifice in its center is subject to a progressively increasing forced displacement in its top border, while the displacement in the bottom border is set to zero. The material properties are the following: the Young modulus is set to $E=10 \mathrm{MPa}$, the Poisson coefficient is $\nu=0.3$, which results in a bulk modulus $\kappa=8.333 \mathrm{MPa}$ and a shear modulus $\mu=3.846 \mathrm{MPa}$. The initial damage 

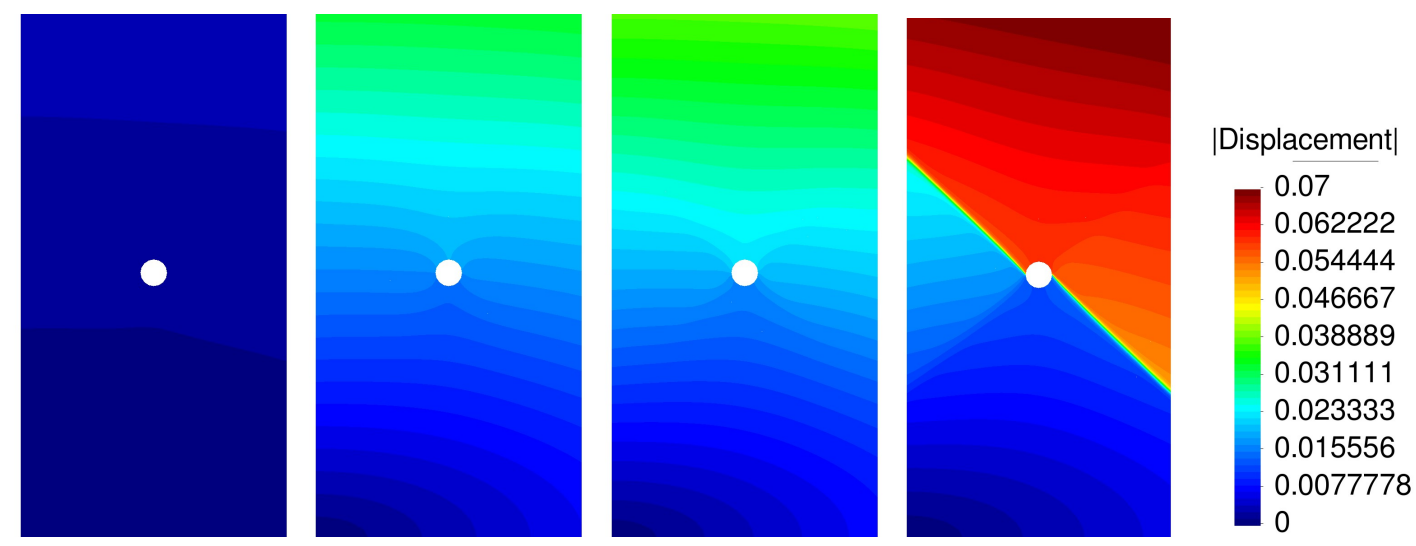

Figure 9: Displacement field after 5, 64, 75 and 125 simulation steps.
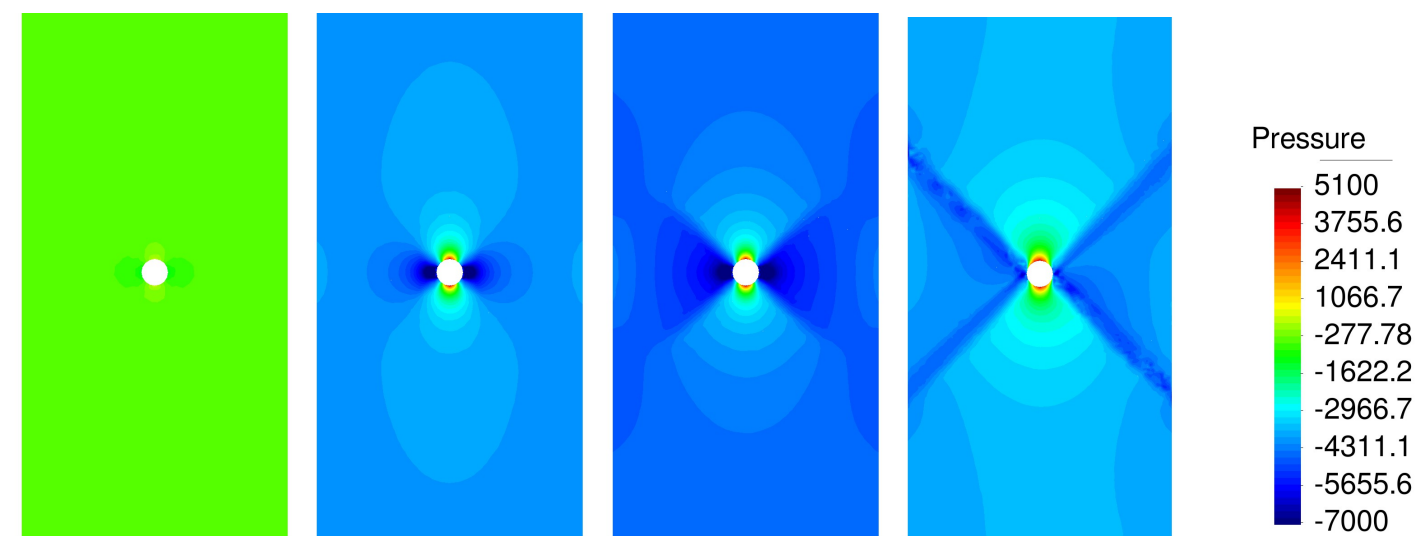

Figure 10: Pressure field after 5, 64, 75 and 125 simulation steps.

uniaxial stress is taken as $r_{0}=10 \mathrm{kPa}$. Mode II fracture energy is taken as $\mathcal{G}_{I I}=200 \mathrm{~J} / \mathrm{m}^{2}$. Again, linear triangles are used to discretize the computational domain.

As the stress module increases, the material gets damaged, damage starts first in the region surrounding the central orifice. Finally a crack appears departing from the central orifice and propagating with an angle of $3 \pi / 4$. Figs. 9 and 10 show the displacement and pressure history as the simulation evolves. As the displacement increases large strains appear in the region surrounding the central orifice. The $3 \pi / 4$ oriented crack can be observed in the last plot. Although initially (due to the simmetry of both loads and geometry) a second crack starts to develop with an orientation of $\pi / 4$, this crack does not finally grow because the stress in the region decreases once the first crack develops. Which of both cracks finally develops depends on the initial imperfections of the considered problem, which in this case correspond to the lack of symmetry of the finite element mesh used to solve the problem. Fig. 11 shows the spatial distribution of the damage parameter at several simulation steps. In the initial steps the distribution of damage is symmetric, but at the end damage is much larger in the $3 \pi / 4$ direction where the prevailing crack appears. Finally, Fig. 12 shows the adaptive mesh at each of the simulation steps, which has been obtained by using the adaptive refinement criteria based on the orthogonal subgrid scales model for the energy error. The mesh follows the path of the crack, which allows us to conclude that the orthogonal subgrid scales are a suitable error estimator for non-linear constitutive laws for solid mechanics which lead to the apparition of cracks. 

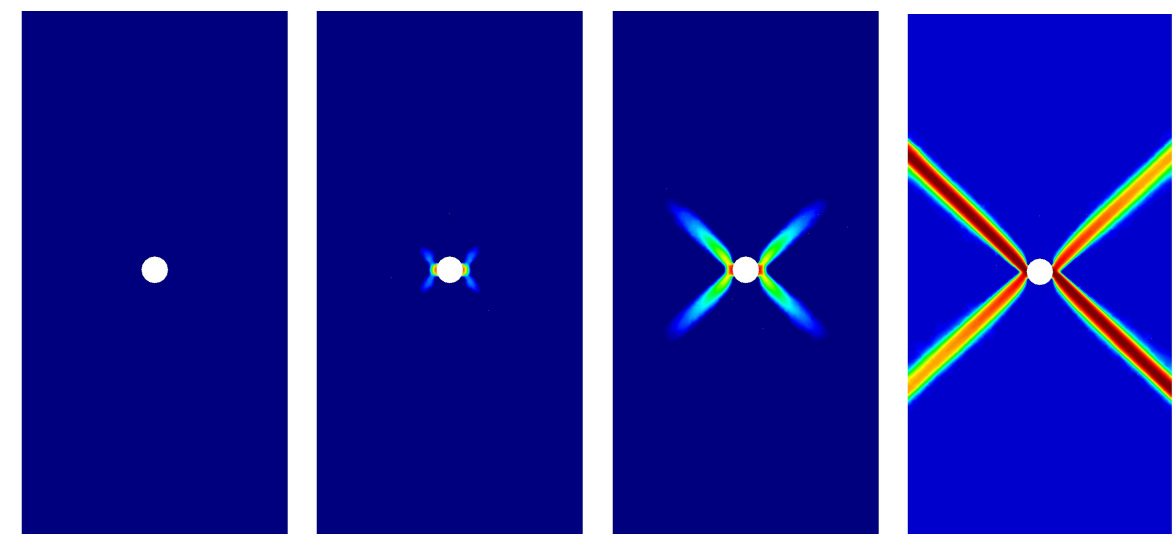

Damage

1
0.87974

0.75948

0.63922

0.51896

0.3987

0.27844

0.15818

0.037916

Figure 11: Damage parameter field after 5, 64, 75 and 125 simulation steps.
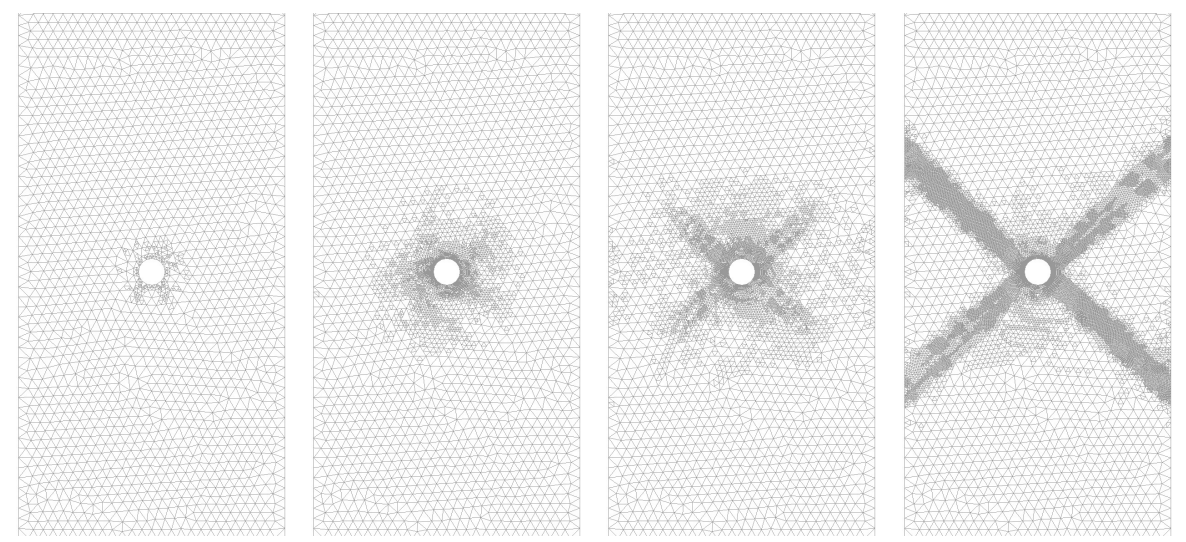

Figure 12: Finite element mesh 5, 64, 75 and 125 simulation steps. 


\subsection{A complex geometry case}

In order to illustrate the capability of the proposed methodology to solve complex problems, in this example we apply the damage model described in Section 4.5 to a case with a complex geometry. The considered geometry is depicted in Fig. 13; the total length of the simulated piece is $8 \mathrm{~cm}$. It consists in a machinery piece with several orifices. The material properties are the following: the Young modulus is set to $E=24 \mathrm{MPa}$, the Poisson coefficient is $\nu=0.21$, which results in a bulk modulus $\kappa=13.79 \mathrm{MPa}$ and a shear modulus $\mu=9.92 \mathrm{MPa}$. The initial damage uniaxial stress is taken as $r_{0}=5 \mathrm{kPa}$. Mode II fracture energy is taken as $\mathcal{G}_{I I}=240 \mathrm{~J} / \mathrm{m}^{2}$. The boundary conditions are depicted in Fig. 14: one of the surfaces of the machinery piece is prescribed null displacements, while another one is prescribed an horizontal displacement. These boundary conditions cause a bending moment to appear in the sections close to the fixed surface. If the bending moment is large enough, it leads to the failure of the material. A mesh of linear tetrahedra is used to discretize the computational domain.

As in the previous example, we are interested in showing that the subgrid scales based error estimator is capable of tracking the apparition of cracks. Fig. 15 shows the evolution of the damage parameter in one of the most stressed areas of the machinery piece, together with the evolution of the finite element mesh as the simulation evolves. The OSS error estimator is capable of tracking and capturing the apparition of damage (which induces the apparition of large deformation gradients), and thus it is suitable to be used for mesh adaptation in complex non-linear materials and geometries.

\section{Conclusions}

In this work we have presented an Orthogonal Subgrid Scale (OSS) [30, 27] error estimator for solid mechanics problems. The OSS error estimator belongs to the family of Variational Multiscale (VMS) based error estimators, with the particularity that the subgrid scales are considered to belong to a space orthogonal to the finite element approximation space. When applied to solid mechanics problems, the error analysis shows that a sharper upper bound can be obtained for the OSS error estimator than for other VMS error estimators such as the Algebraic Subgrid Scale (ASGS) error estimator. Also, the proposed error estimator makes use of a recently developed approximation for the subscales on the element boundaries [28].

The numerical results show that the proposed error estimator is capable of recovering the energy error norm in a variety of situations where the contribution of each of the terms of the error estimator are evaluated: these situations correspond to compressible and incompressible materials, hydrostatic and deviatoric stress states.

Finally the developed error estimator is applied to non-linear solid mechanics problems featuring a non-linear damage constitutive law. In these problems, the proposed error estimator is capable of tracking the apparition of cracks and large concentrated gradients, adding the required mesh refinement in the appropriate areas of the computational domain.

In summary, the OSS error estimator is a suitable estimator for the solution of adaptive finite elements in the context of complex solid mechanics problems.

\section{Acknowledgements}

The authors would like to thank Fermín Otero for providing the geometrical data for the machinery piece example. This work is partially funded through the ELASTIC-FLOW project, Ref. DPI2015-67857-R of the Spanish Government. J. Baiges acknowledges the support of 

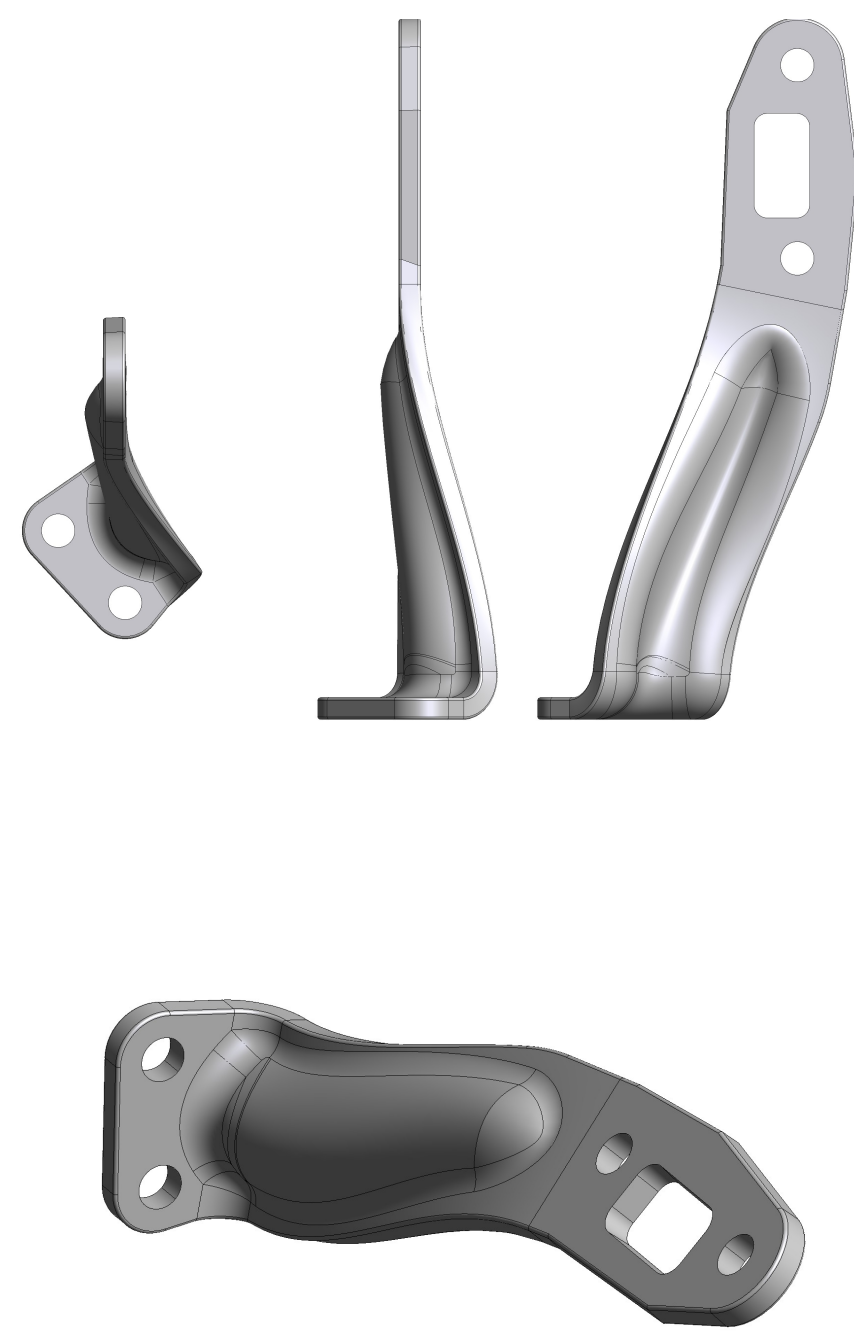

Figure 13: Complex geometry case. Top: $x y, x z$ and $y z$ views. Bottom: free view of the geometry

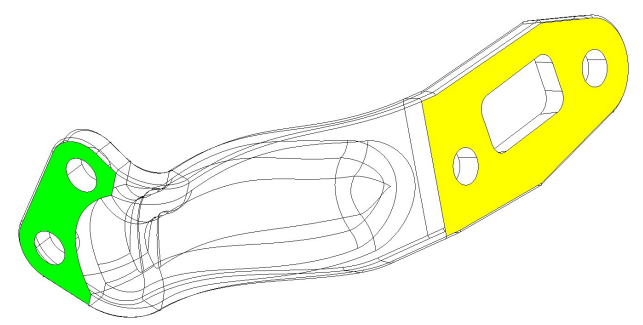

Figure 14: Boundary conditions for the complex geometry case. The green surface is prescribed null displacements in all directions. The yellow surface is prescribed an increasing displacement in the $x$ direction. 

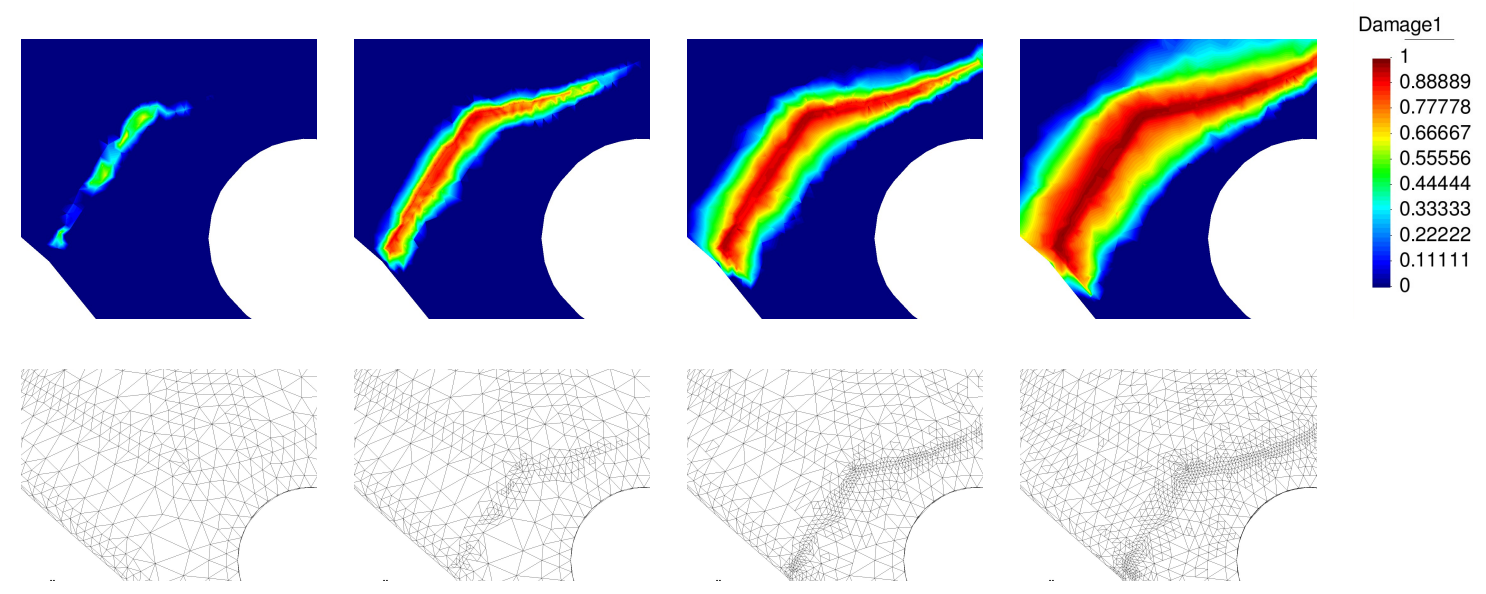

Figure 15: Damage parameter evolution and mesh evolution in the most stressed areas of the machinery piece.

the Spanish Government through the Ramón y Cajal grant RYC-2015-17367. R. Codina acknowledges the support received through the ICREA Acadèmia Research Program of the Catalan Government. The support of the European PRACE computational network and the RES-Barcelona Supercomputing center in the development of the distributed memory adaptive mesh refinement algorithms is acknowledged. The financial support to CIMNE via the CERCA Programme / Generalitat de Catalunya is also acknowledged.

\section{References}

[1] Verfürth R. A review of a posteriori error estimation and adaptive mesh-refinement techniques. John Wiley \& Sons Inc; 1996.

[2] Vanella M, Posa A, Balaras E. Adaptive mesh refinement for immersed boundary methods. Journal of Fluids Engineering. 2014;136(4):040909.

[3] Russell RD. Adaptive Mesh Refinement. Encyclopedia of Applied and Computational Mathematics. 2015;p. 23-25.

[4] Badia S, Baiges J. Adaptive finite element simulation of incompressible flows by hybrid continuous-discontinuous Galerkin formulations. SIAM Journal on Scientific Computing. 2013;35(1):A491-A516.

[5] Baiges J, Bayona C. RefficientLib: An efficient load-rebalanced adaptive mesh refinement algorithm for high performance computational physics meshes. Submitted. 2015;

[6] Baiges J, Codina R, Pont A, Castillo E. An adaptive Fixed-Mesh ALE method for free surface flows. Computer Methods in Applied Mechanics and Engineering. 2017;313:159 - 188. Available from: http://www.sciencedirect.com/science/ article/pii/S0045782516312567.

[7] Ainsworth M, Oden JT. A Posteriori Error Estimation in Finite Element Analysis. Wiley-Interscience; 2000.

[8] Verfürth R. A posteriori error estimation techniques for finite element methods. Oxford University Press; 2013. 
[9] Hughes TJR. Multiscale phenomena: Green's function, the Dirichlet-to-Neumann formulation, subgrid scale models, bubbles and the origins of stabilized formulations. Computer Methods in Applied Mechanics and Engineering. 1995;127:387-401.

[10] Hughes TJR, Feijóo GR, Mazzei L, Quincy JB. The variational multiscale method-a paradigm for computational mechanics. Computer Methods in Applied Mechanics and Engineering. 1998;166:3-24.

[11] Hughes TJ, Scovazzi G, Franca LP. Multiscale and stabilized methods. Encyclopedia of computational mechanics. 2004;

[12] Codina R, Badia S, Baiges J, Principe J. Variational Multiscale Methods in Computational Fluid Dynamics. Encyclopedia of computational mechanics. 2017;

[13] Hauke G, Doweidar MH, Miana M. The multiscale approach to error estimation and adaptivity. Computer methods in applied mechanics and engineering. 2006;195(13):15731593.

[14] Hauke G, Doweidar M, Fuster D, Gómez A, Sayas J. Application of Variational a-Posteriori Multiscale Error Estimation to Higher-Order Elements. Computational Mechanics. 2006;38(4-5):382-389. Available from: http://dx.doi.org/10.1007/ s00466-006-0048-7.

[15] Hauke G, Doweidar MH, Miana M. Proper intrinsic scales for a-posteriori multiscale error estimation. Computer methods in applied mechanics and engineering. 2006;195(33):3983-4001.

[16] Hauke G, Fuster D, Doweidar MH. Variational multiscale a-posteriori error estimation for multi-dimensional transport problems. Computer Methods in Applied Mechanics and Engineering. 2008;197(33):2701-2718.

[17] Rossi R, Cotela J, Lafontaine NM, Dadvand P, Idelsohn S. Parallel adaptive mesh refinement for incompressible flow problems. Computers \& Fluids. 2013;80:342-355.

[18] Hauke G, Fuster D, Lizarraga F. Variational multiscale a posteriori error estimation for systems: The Euler and Navier-Stokes equations. Computer Methods in Applied Mechanics and Engineering. 2015;283:1493-1524.

[19] Hauke G, Irisarri D. Variational multiscale a posteriori error estimation for systems. Application to linear elasticity. Computer Methods in Applied Mechanics and Engineering. 2015;285:291-314.

[20] Irisarri D, Hauke G. Variational multiscale a posteriori error estimation for 2nd and 4th-order ODEs. International Journal of Numerical Analysis \& Modeling. 2015;12(3).

[21] Masud A, Truster TJ, Bergman LA. A variational multiscale a posteriori error estimation method for mixed form of nearly incompressible elasticity. Computer Methods in Applied Mechanics and Engineering. 2011;200(47):3453-3481.

[22] John V, Novo J. A robust SUPG norm a posteriori error estimator for stationary convection-diffusion equations. Computer Methods in Applied Mechanics and Engineering. 2013;255:289-305. 
[23] Larson MG, Målqvist A. Adaptive variational multiscale methods based on a posteriori error estimation: energy norm estimates for elliptic problems. Computer methods in applied mechanics and engineering. 2007;196(21):2313-2324.

[24] Hou TY, Wu XH. A multiscale finite element method for elliptic problems in composite materials and porous media. Journal of Computational Physics. 1997;134(1):169-189.

[25] Larson MG, Målqvist A. An adaptive variational multiscale method for convectiondiffusion problems. Communications in Numerical Methods in Engineering. 2009;25(1):65-79.

[26] Målqvist A, Peterseim D. Localization of elliptic multiscale problems. Mathematics of Computation. 2014;83(290):2583-2603.

[27] Codina R. Stabilized finite element approximation of transient incompressible flows using orthogonal subscales. Computer Methods in Applied Mechanics and Engineering. 2002;191:4295-4321.

[28] Codina R, Principe J, Baiges J. Subscales on the element boundaries in the variational two-scale finite element method. Computer Methods in Applied Mechanics and Engineering. 2009;198:838-852.

[29] Codina R, Baiges J. Finite element approximation of transmission conditions in fluids and solids introducing boundary subgrid scales. International Journal for Numerical Methods in Engineering. 2011;87:386-411.

[30] Codina R. A stabilized finite element method for generalized stationary incompressible flows. Computer Methods in Applied Mechanics and Engineering. 2001;190:2681-2706.

[31] Codina R, Principe J, Guasch O, Badia S. Time dependent subscales in the stabilized finite element approximation of incompressible flow problems. Computer Methods in Applied Mechanics and Engineering. 2007;196:2413-2430.

[32] Castillo E, Baiges J, Codina R. Approximation of the two-fluid flow problem for viscoelastic fluids using the level set method and pressure enriched finite element shape functions. Journal of Non-Newtonian Fluid Mechanics. 2015;225:37 - 53. Available from: http://www.sciencedirect.com/science/article/pii/S0377025715001688.

[33] Codina R, Blasco J. A finite element formulation for the Stokes problem allowing equal velocity-pressure interpolation. Computer Methods in Applied Mechanics and Engineering. 1997;143:373-391.

[34] Codina R. Stabilization of incompressibility and convection through orthogonal subscales in finite element methods. Computer Methods in Applied Mechanics and Engineering. 2000;190:1579-1599.

[35] El-Alaoui L, Ern A. Residual and Hierarchical a posteriori error estimates for nonconforming mixed finite element methods. ESAIM: Mathematical Modelling and Numerical Analysis. 2004;38(6):903-929.

[36] Ainsworth M, Oden JT. A posteriori error estimators for the Stokes and Oseen equations. SIAM Journal on Numerical Analysis. 1997;34:228-245.

[37] Ern A, Guermond JL. Theory and Practice of Finite Element. Springer-Verlag; 2004. 
[38] Codina R, Principe J, Ávila M. Finite element approximation of turbulent thermally coupled incompressible flows with numerical sub-grid scale modelling. International Journal of Numerical Methods for Heat \& Fluid Flow. 2010;20(5):492-516.

[39] Cervera M, Chiumenti M, Agelet de Saracibar C. Shear band localization via local J2 continuum damage mechanics. Computer Methods in Applied Mechanics and Engineering. 2004;193:849-880. 